\title{
ANION-SELECTIVE MEMBRANE ELECTRODES BASED ON METALLOPORPHYRINS: THE INFLUENCE OF LIPOPHILIC ANIONIC AND CATIONIC SITES ON POTENTIOMETRIC SELECTIVITY
}

\author{
Eric Bakker, Elzbieta Malinowska, * Robert D. Schiller and Mark E. Meyerhoff† \\ Department of Chemistry, The University of Michigan, Ann Arbor, MI 48109, U.S.A.
}

(Received 31 December 1993. Revised 18 January 1994. Accepted 19 January 1994)

\begin{abstract}
Summary-The role of lipophilic anionic and cationic additives on the potentiometric anion selectivities of polymer membrane electrodes prepared with various metalloporphyrins as anion selective ionophores is examined. The presence of lipophilic anionic sites (e.g. tetraphenylborate derivatives) is shown to enhance the non-Hofmeister anion selectivities of membranes doped with In(III) and Sn(IV) porphyrins. In contrast, membranes containing $\mathrm{Co}(\mathrm{III})$ porphyrins require the addition of lipophilic cationic sites (e.g. tridodecylmethylammonium ions) in order to achieve optimal anion selectivity (for nitrite and thiocyanate) as well as rapid and reversible Nernstian response toward these anionic species. These experimental results coupled with appropriate theoretical models that predict the effect of lipophilic anion and cation sites on the selectivities of membranes doped with either neutral or charged carrier type ionophores may be used to determine the operative ionophore mechanism of each metalloporphyrin complex within the organic membrane phase.
\end{abstract}

Among an increasing variety of anion-selective ionophores described recently for preparation of solvent polymeric membrane electrodes ${ }^{1-3}$ metalloporphyrins have emerged as particularly interesting species in that they yield anion sensors with selectivity patterns that deviate significantly from the classical Hofmeister sequence. The unique potentiometric selectivities observed with membranes doped with these metal-ligand complexes is thought to result from the selective interaction (ligation) of given analyte anions with the metal center of the porphyrin structure within the organic membrane phase. Accordingly, use of porphyrins with different metal centers lead to sensors with distinctly different preferences for given anions. For example, use of $\mathrm{Mn}(\mathrm{III}),{ }^{4-6} \mathrm{Sn}(\mathrm{IV}),{ }^{7,8} \mathrm{In}(\mathrm{III}),{ }^{9} \mathrm{Ru}(\mathrm{II})^{10}$ and $\mathrm{Co}(\mathrm{III})^{4,10-12}$ porphyrins have led to the design of a variety of anion sensors with analytically useful selectivities for thiocyanate, salicylate, chloride, thiocyanate and nitrite/thiocyanate, respectively.

It has been recognized previously that anion ionophores acting as neutral carriers within the

*On leave from the Department of Analytical Chemistry, Warsaw Technical University, Poland. †Author to whom correspondence should be addressed. membrane phase (i.e. electrically neutral when not complexed, and negatively charged when complexed to the target anion) need the simultaneous incorporation of lipophilic cationic additives to induce appropriate anionic response slopes and optimum potentiometric selectivity. Indeed, the presence of endogenous anionic impurities in poly(vinyl chloride), ${ }^{13,14}$ the matrix most often used to prepare functional ion sensors, would otherwise hinder the anion response and lead to sensors with poor potentiometric response characteristics. However, to date, compounds believed to function as charged carrier type ionophores (i.e. ionophore is positively charged in native form and neutral when complexed to target anion) have been used without the addition of exogenous lipophilic ionic sites, since the observed slopes were clearly anionic and the membrane selectivities quite promising. In previous work, metalloporphyrin ionophores that induce a non-Hofmeister potentiometric selectivity in polymeric membranes without addition of cationic sites were assumed to function as charged-carriers, whereas those that operate presumably via a neutral carrier mechanism required the incorporation of lipophilic quaternary ammonium salts to obtain appropriate response characteristics. ${ }^{10}$ Only very recently 
has the role of lipophilic anionic sites on the response properties of membranes doped with electrically charged anion-selective ionophores been considered, both theoretically and experimentally, using $\mathrm{Co}$ (III) cobyrinates as model charged carrier ionophores. ${ }^{\text {Is }}$ This recent work has important implications with respect to the operative mechanism of all charged carrierbased membrane electrodes, and is especially interesting for the evaluation of selectivity of membranes containing metalloporphyrins, since some of these carriers could act equally as neutral or charged carrier type ionophores (see Fig. 1).

In this work, we underline the importance of ionic additives as well as endogenous ionic impurities on the potentiometric selectivities of membranes containing porphyrins as membrane ionophores. It is shown that the selectivities of membranes containing Sn(IV) tetraphenyl and In(III) octaethyl porphyrin are clearly improved by the incorporation of tetraphenylborate derivatives as anionic sites, whereas $\mathrm{Co}$ (III) tetraphenylporphyrin appears to function as a neutral carrier, requiring quaternary ammonium salts to induce a Nernstian anionic slope and nonHofmeister selectivity toward nitrite and thiocyanate.

\section{EXPERIMENTAL}

\section{Reagents}

The ionophores dichloro(5,10,15,20-tetraphenylporphyrinato)tin(IV) $\quad\left(\mathrm{Sn}[\mathrm{TPP}] \mathrm{Cl}_{2}\right),{ }^{16}$ chloro $(2,3,7,8,12,13,17,18$ octaethyl porphyrinato) indium(III) (In(III)[OEP]Cl), ${ }^{16}$ and chloro $(5,10,15,20$-tetraphenylporphyrinato)cobalt(III) (Co[TPP]Cl), ${ }^{17,18}$ were synthesized according to previously published procedures. For polymer membrane preparation, $o$-nitrophenyloctylether $(o$-NPOE), potassium tetrakis $\{$ bis $(m$-trifluoromethyl)phenyl\}borate (KTFPB), tridodecylmethylammonium chloride (TDMACl), poly(vinyl chloride) (PVC, high mol. weight), tetrahydrofuran (THF, distilled prior to use) were purchased from Fluka (Ronkonkoma, NY).

All aqueous solutions were prepared with salts and acids of the highest purity available. The sample solutions for all potentiometric measurements consisted of sodium salts of the indicated anions in 0.05M 4-morpholinoethanesulfonic acid (MES), adjusted to $\mathrm{pH} 5.5$ with $\mathrm{NaOH}$.

\section{ISE membranes and EMF measurements}

The ion-selective electrode membranes containing $0.6-1 \quad w t \%$ ionophore and different amounts of ionic additives (KTFPB or TDMACl) in $o$-NPOE/PVC $(2: 1)$ were prepared as described previously. ${ }^{19}$ The potentiometric measurements were performed with the following galvanic cell: $\mathrm{Ag} / \mathrm{AgCl} /$ bridge electrolyte/sample/ion-selective membrane/inner filling solution/AgCl/Ag. The bridge electrolyte consisted of $0.1 M \mathrm{KNO}_{3}$ [for the measurements of membranes with $\mathrm{Co}$ (III) tetraphenylporphyrin] or $0.05 \mathrm{M}$ MES buffer, pH 5.5 (for the other porphyrins). The inner filling solution for membranes containing $\mathrm{In}(\mathrm{III}) \mathrm{OEPCl}$ or TDMACl alone was $0.01 \mathrm{M} \mathrm{NaCl}$, while for $\mathrm{Co}$ (III)TPPCl-based membranes, $0.01 \mathrm{M}$ $\mathrm{NaNO}_{2} / 0.1 M \mathrm{NaCl}$ solution was used. In the case of electrodes prepared with membranes doped with $\mathrm{Sn}$ (IV)TPPCl ${ }_{2}$, a $10^{-4} \mathrm{M}$ sodium salicylate/0.01M NaCl was used. Prior to EMF measurements, the electrodes were conditioned overnight in a solution having the same composition as the inner filling solution.

All experiments were performed at ambient temperature $\left(22 \pm 2^{\circ} \mathrm{C}\right)$. Potentials were measured using a Macintosh IIcx computer with an NB-MIO-16X analog/digital input/ output board (National Instruments, Austin, TX) and a custom built electrode interface module controlled by Lab-View 2 software (National In-

A)

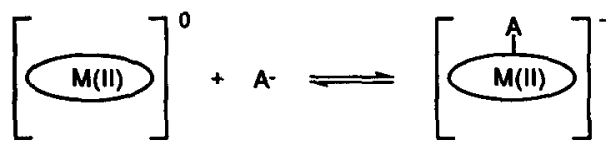

B)

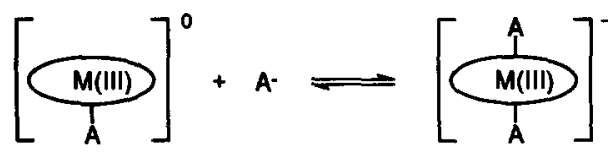

C)

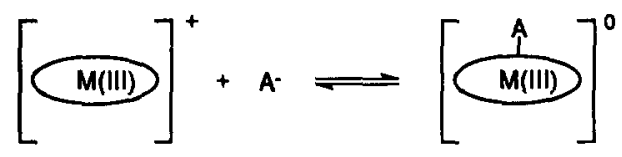

D)

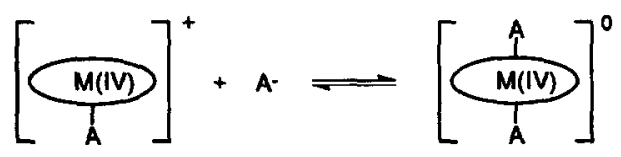

Fig. 1. Influence of the metal center oxidation state on the possible anion-ionophore interactions. Charged axial ligands are denoted with (A), neutral ligands are not shown. The metal(III) porphyrins may act as electrically neutral (B) or charged carriers (C) depending on the charge of the fifth axial ligand. 
struments) as described previously. ${ }^{20}$ Selectivity coefficients were determined according to the separate solution method ${ }^{21}$ by using the experimental EMF values for $0.01 M$ solutions of the test anions and a theoretical slope of $-59.2 \mathrm{mV} / \mathrm{dec}$ for the primary anion. Activity coefficients were assumed to be constant for all analyte anions, and no correction was made for slight changes in the liquid junction potential of the reference electrode.

\section{THEORETICAL CONSIDERATIONS}

As shown in Fig. 1, metalloporphyrins may act as electrically neutral or electrically charged carriers within the membrane phase, depending on the oxidation state of the metal center and the number of coordinated anionic axial ligands. Hence, significantly different potentiometric behavior of electrodes prepared with varying porphyrins should be expected depending on the complexation mechanism involved. Below, we summarize the predicted effect of adding lipophilic electrically charged additives to the membranes on the selectivity of electrodes containing carriers that operate via either of these two mechanisms.

\section{Membranes with electrically neutral carriers}

In contrast to cation-selective electrodes, divalent interfering ions (e.g. $\mathrm{CO}_{3}^{2-}, \mathrm{SO}_{4}^{2-}$, $\mathrm{HPO}_{4}^{2-}$, etc.) have been shown to exhibit relatively little interference for metalloporphyrinbased anion-selective electrodes. ${ }^{4} \mathrm{It}$ is, therefore, appropriate to investigate the selectivity of anion sensors with respect to monovalent interferent anions only. It is further assumed that, if any, only $1: 1$ complexes of the anion with the metalloporphyrin ionophore $L$ are formed, and the fifth ligand bound to the metal center can be regarded as non-exchangeable under the experimental conditions tested (see Fig. 1). Thus, to determine the amount of cationic additives relative to the ionophore concentration required to achieve optimal selectivity, the following general formalism for the Nicolsky selectivity coefficient, $\log k_{\mathrm{XY}}^{\mathrm{pot}}$, is used: $:^{15}$

$$
\log k_{\mathrm{XY}}^{\mathrm{oot}}=\log K_{\mathrm{XY}}+\log \frac{\left[\mathrm{X}^{-}\right]}{\left[\mathrm{Y}^{-}\right]},
$$

with $K_{\mathrm{XY}}$ being the ion-exchange equilibrium constant for the so-called free primary and interfering ion $X$ and $Y$, respectively

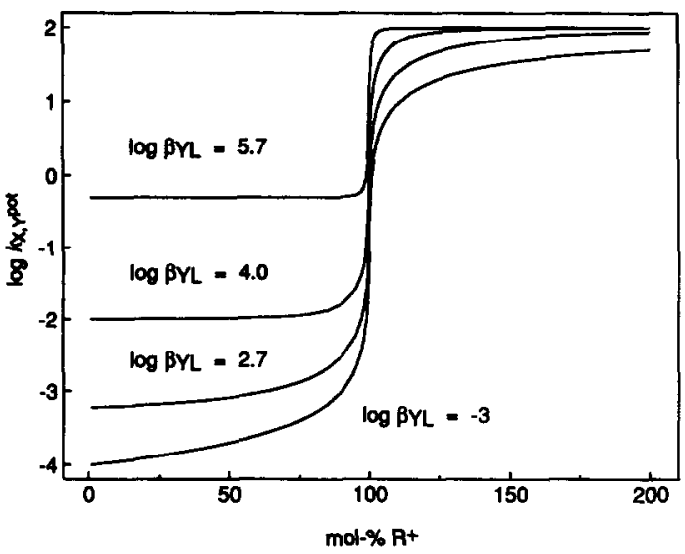

Fig. 2. Calculated selectivity coefficients according to equation 2 for a membrane containing a neutral carrier as a function of the concentration of cationic sites in the membrane $\left(L_{\mathrm{T}}=0.01 \mathrm{~mol} / \mathrm{kg}, \log K_{\mathrm{XY}}=2, \log \beta_{\mathrm{XL}}=8\right)$.

$\left(K_{\mathrm{XY}}=a_{\mathrm{Y}} *\left[\mathrm{X}^{-}\right] /\left(a_{\mathrm{X}} *\left[\mathrm{Y}^{-}\right]\right)\right)$, and $\left[\mathrm{X}^{-}\right]$and $\left[\mathrm{Y}^{-}\right]$ are the concentration of uncomplexed primary and interfering anions, respectively, in the organic phase boundary contacting the aqueous sample solution. These two values are obtained from two different experiments, each solution containing the salt of one anion alone. Accordingly, electroneutrality conditions $\left(R_{T}^{+}=\right.$ $\left[\mathrm{X}^{-}\right]+\left[\mathrm{XL}^{-}\right]$and $\left.R_{\mathrm{T}}^{+}=\left[\mathrm{Y}^{-}\right]+\left[\mathrm{YL}^{-}\right]\right)$and mass balances for the ionophore $\left(L_{\mathrm{T}}=[\mathrm{L}]+\left[\mathrm{XL}^{-}\right]\right.$and $\left.L_{\mathrm{T}}=[\mathrm{L}]+\left[\mathrm{YL}^{-}\right]\right)$can be applied for both experimental cases $\left(L_{\mathrm{T}}\right.$ and $R_{\mathrm{T}}^{+}$ designate the total concentration of ionophore and cationic additive, respectively). After inserting these conditions into equation 1 , together with the definitions of the complex stability constants $(\beta)$, the following expression for the selectivity coefficient is obtained:

$$
\begin{aligned}
& k{ }_{\mathrm{XY}}^{\mathrm{pot}}=K_{\mathrm{XY}} \frac{\beta_{\mathrm{YL}}}{\beta_{\mathrm{XL}}} . \\
& \frac{\beta_{\mathrm{XL} \cdot} \cdot\left(L_{\mathrm{T}}-R_{\mathrm{T}}^{+}\right)+\sqrt{\beta_{\mathrm{XL}}^{2} \cdot\left(L_{\mathrm{T}}-R_{\mathrm{T}}^{+}\right)^{2}+4 \beta_{\mathrm{XL}} R_{\mathrm{T}}^{+}}}{\beta_{\mathrm{YL} \cdot} \cdot\left(L_{\mathrm{T}}-R_{\mathrm{T}}^{+}\right)+\sqrt{\beta_{\mathrm{YL}}^{2} \cdot\left(L_{\mathrm{T}}-R_{\mathrm{T}}^{+}\right)^{2}+4 \beta_{\mathrm{YL}} R_{\mathrm{T}}^{+}}},
\end{aligned}
$$

where $\beta_{\mathrm{YL}}$ and $\beta_{\mathrm{XL}}$ denote the respective formation constants for the complexes of anions $\mathrm{X}^{-}$and $\mathrm{Y}^{-}$with the ionophore $\mathrm{L}$ in the membrane phase. It should be noted that equation 2 is only valid under the assumption that the diffusion potential within the membrane phase and any ion pair formation with the lipophilic ionic additive is negligible. The effect of such ion pair formation on the selectivity of cation-selective electrodes has been described previously. ${ }^{22}$

In Fig. 2, theoretically predicted selectivity 
coefficients (from equation 2) as a function of the concentration of cationic additives in the membrane are shown. For monovalent ions forming no complexes with the ionophore (e.g. $\beta_{\mathrm{YL}}=10^{-3}$ ), the optimum composition is achieved at a low concentration of $R_{T}^{+}$. However, interfering ions forming stable complexes with the ionophore induce no appreciable change in the selectivity coefficient as a function of $R_{\mathrm{T}}^{+}$, and the following expression holds: ${ }^{23}$

$$
\log k \underset{\mathrm{XY}}{\text { ot }}=\log K_{\mathrm{XY}}+\log \frac{\beta_{\mathrm{YL}}}{\beta_{\mathrm{XL}}} .
$$

This expression will only be true for a membrane with less than $100 \mathrm{~mol} \%$ cationic additive relative to the total ionophore concentration (see Fig. 2). With higher concentrations, the selectivity will be governed by the non-specific anion-exchange capability of the cationic additive alone. Therefore, the optimum composition of neutral carrier-based membrane will usually be achieved with a low amount of cationic additives, which must be found experimentally. Previously, about $2 \mathrm{~mol} \%$ of $R_{\mathrm{T}}^{+}$have been shown to be sufficient to overcome the naturally present anionic impurities ${ }^{13,14}$ of poly(vinyl chloride) membranes and induce permselectivity for anions. ${ }^{3}$

Figure $3(\mathrm{~A}-\mathrm{C})$ schematically illustrates the equilibrium compositions of membranes containing anionic, cationic, or no additives for the case when the metalloporphyrin ionophore is functioning as a neutral carrier. Lipophilic cationic additives form the counterions of the

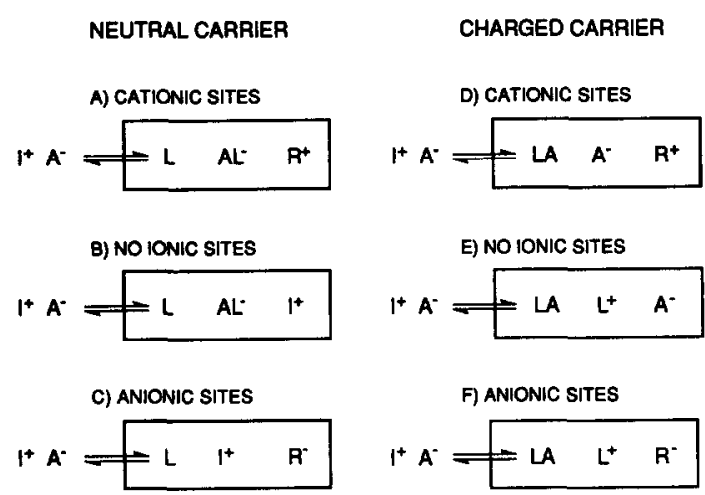

Fig. 3. Schematic presentation of charged and neutral carrier mechanisms with and without ionic additives in the membrane phase. The boxes designate the organic phase, $\mathrm{A}^{-}$and $\mathrm{I}^{+}$the sample ions, $\mathrm{L}$ the ionophore and $\mathrm{R}^{-} / \mathrm{R}^{+}$the lipophilic ionic additive of the respective charge type. Optimum selectivity according to equation 3 is only achieved in cases (A) and (F), where the amount of ionic additive controls the ratio of free to complexed ionophore in the membrane phase. negatively charged complexes in the membrane phase, and therefore, the ratio of uncomplexed to complexed ionophore is held constant for monovalent ions forming stable $1: 1$ complexes with the ionophore (see Fig. 3A).

However, if no lipophilic ionic species are present in the membrane phase, the amount of complexes formed is given solely by the following co-extraction equilibrium process (see Fig. 3B):

$$
\begin{aligned}
\mathrm{X}^{-}(\mathrm{aq})+\mathrm{I}^{+}(\mathrm{aq})+ & \mathrm{L}(\mathrm{org}) \\
& \rightleftharpoons \mathrm{XL}^{-} \text {(org) }+\mathrm{I}^{+} \text {(org), }
\end{aligned}
$$

where aq and org denote aqueous and organic phases, respectively. Hence, the counterions are formed by the extracted cations from the aqueous phase. However, in the activity range where the concentration of uncomplexed ionophore $\mathrm{L}$ in the membrane remains essentially constant, the co-extraction process does not induce a change in the phase boundary potential at the sample/membrane interface. ${ }^{24}$ Consequently, the observed electrode function will likely be sub-Nernstian and analytically not useful.

The incorporation or presence of lipophilic anionic species in a membrane containing a neutral anion-selective carrier is depicted schematically in Fig. 3C. Since neither the negatively charged ionophore complex nor the neutral carrier can act as the positive counterion of the additive, the cations present in the sample have to be extracted into the membrane to maintain electroneutrality in the membrane. Therefore, the electrode will no longer respond to anions, and a Nernstian response slope to cations in the sample solution is expected.

\section{Membranes with electrically charged carriers}

A detailed theoretical discussion of the selectivity behavior of charged-carrier based ion-selective electrodes has been presented elsewhere. ${ }^{15}$ Therefore, only the most important results of this previous work as they relate to the use of metalloporphyrins as ionophores is presented here.

As shown in Fig. 3(D-F), the anion complexes of charged carriers are electrically neutral when monovalent primary ions complexing with a 1:1 stoichiometry with the ionophore are present. If no lipophilic ionic additives are introduced or are naturally present in the membrane phase (due to impurities in the polymer matrix), the amount of uncomplexed ionophore and 
analyte ion in the membrane is given by the respective dissociation constant of the complex (see Fig. 3E). If an interfering ion of the same charge is extracted into the membrane and complexed by the ionophore, the amount of uncomplexed interfering ion in the membrane will be higher than the primary ion, if it forms a weaker complex with the ionophore. Therefore, by considering the respective mass balances for the ionophore and electroneutrality condition for the membrane phase, one obtains the following expression for the potentiometric selectivity coefficient: ${ }^{15}$

$$
\log k \text { XY } \text { 吕 }=\log K_{\mathrm{XY}}+\frac{1}{2} \log \frac{\beta_{\mathrm{YL}}}{\beta_{\mathrm{XL}}} .
$$

Hence, when compared to equation 3 , less than optimum selectivity is expected for charged-carrier based electrodes without addition of lipophilic anionic sites. This is an indication that the selectivity of pure liquid membrane electrodes containing an electrically charged ionophore only ${ }^{6}$ is not solely that predicted based on the relative anion complexation constants.

In contrast, lipophilic additives of negative charge will form the lipophilic counterions of the free, and therefore positively charged carrier in the membrane. Consequently, as shown in Fig. 3F, these additives define the ratio of complexed to uncomplexed ionophore in the membrane phase. This ratio will be equal for any interfering ion having the same charge and forming complexes of equal stoichiometry as the primary ion. Again, after consideration of mass balance for the ionophore and electroneutrality conditions, the selectivity coefficient will be given by equation 3, and this situation corresponds exactly to the case of the neutral carrier and cationic additives shown above.

A completely different situation is presented in Fig. 3D, where cationic additives such as tetralkylammonium salts are incorporated into the membrane containing metalloporphyrins that function as charged carriers. Since the ionophore and its complexes are not negatively charged, these additives will extract uncomplexed anions from the sample into the organic phase. The electrode will still respond to anionic species, but since these are not complexed by the ionophore, a non-specific Hofmeister selectivity behavior is expected as predicted by the following equation:

$$
\log k \underset{\mathrm{XY}}{\text { ol }}=\log K_{\mathrm{XY}} .
$$

Based on the above considerations, a certain minimum amount of lipophilic anionic additives, such as tetraphenylborate derivatives, must be incorporated into membranes which contain a positively charged ionophore in order to achieve electrodes with optimum anion selectivity. More importantly, however, is to recognize that for all three cases shown in Fig. 3(D-F), the resulting sensor would in fact exhibit anionic and potentially useful response characteristics, albeit in some instances (i.e. Fig. 3D and E), less than optimal selectivity. This fact may explain why the importance of ionic additives in the performance of charged carrier-based electrodes has only recently been recognized. ${ }^{15}$

\section{RESULTS AND DISCUSSION}

As stated above, the specific oxidation state of the metal center incorporated in the porphyrin ring structure is expected to have an important influence on the possible carrier mechanism when these species are incorporated into polymer membranes for the purpose of preparing anion-selective sensors. Especially interesting are the results that have been obtained for membranes doped with $\mathrm{Ru}(\mathrm{II}), \mathrm{In}(\mathrm{III}), \mathrm{Sn}(\mathrm{IV})$ and $\mathrm{Co}(\mathrm{III})$ porphyrins which offer unique selectivities for thiocyanate, chloride, salicylate and nitrite/ thiocyanate, respectively. Assuming that these metalloporphyrins have a coordination number of six, the fifth ligand is assumed to be inert and may be neutral (e.g. water; not shown in Fig. 1) or negatively charged (depicted with the symbol A), while the sixth ligand denotes the analyte anion. Therefore, porphyrins with metal(II) centers can only function via a neutral carrier mechanism, ${ }^{10}$ while metal(IV) porphyrins must operate via a charged carrier mechanism. However, a metal(III) center within a given porphyrin structure will enable the ionophore to act as both a neutral or charged carrier, depending on the number of negatively charged axial ligands on the metal center. Therefore, the optimum composition of membranes containing these carriers is not known beforehand and has to be evaluated experimentally. Membranes containing lipophilic cationic or anionic additives should only exhibit an improved selectivity according to equation 3 , if one of the optimum membrane situations depicted in Fig. 3(A or F) is reached. 
Table 1. Selectivity coefficients of $0 . \mathrm{NPOE} / \mathrm{PVC}(2: 1)$ membranes doped with $\mathrm{Sn}(\mathrm{IV})[\mathrm{TPP}] \mathrm{Cl}_{2}$ and different concentrations of ionic additives

\begin{tabular}{|c|c|c|c|c|c|c|}
\hline \multirow[b]{2}{*}{$\mathbf{Y}^{-}$} & \multicolumn{6}{|c|}{$\log k$ Sal,Y } \\
\hline & $30 \% \mathbf{R}^{-}$ & $20 \% \mathbf{R}^{-*}$ & $10 \% \mathrm{R}^{-*}$ & $0 \% *$ & $10 \% \mathrm{R}^{+*}$ & TDMACl$\dagger$ \\
\hline $\begin{array}{l}\mathrm{Cl}^{-} \\
\mathrm{NO}_{2}^{-} \\
\mathrm{NO}_{3}^{-} \\
\mathrm{SCN}^{-} \\
\mathrm{I}^{-} \\
\mathrm{ClO}_{4}^{-}\end{array}$ & $\begin{array}{l}-2.8 \\
-2.3 \\
-2.8 \\
-2.4 \\
-2.8 \\
-2.8\end{array}$ & $\begin{array}{l}-3.0 \\
-2.4 \\
-2.8 \\
-2.5 \\
-2.9 \\
-2.9\end{array}$ & $\begin{array}{l}-4.2 \\
-3.1 \\
-3.8 \\
-2.9 \\
-4.0 \\
-3.6\end{array}$ & $\begin{array}{l}-2.2 \\
-2.0 \\
-2.4 \\
-1.0 \\
-1.9 \\
-0.9\end{array}$ & $\begin{array}{r}-2.3 \\
-2.4 \\
-1.6 \\
0.0 \\
-0.2 \\
1.2\end{array}$ & $\begin{array}{r}-3.3 \\
-2.4 \\
-1.6 \\
0.0 \\
0.0 \\
1.4\end{array}$ \\
\hline
\end{tabular}

*Percentage given: molar amount of $\mathrm{KTFPB}\left(\mathrm{R}^{-}\right)$or $\mathrm{TDMACl}\left(\mathrm{R}^{+}\right)$relative to the total ionophore concentration in the membrane.

+Membrane with $1 \mathrm{wt} \%$ TDMACl (without ionophore).

Metalloporphyrins as electrically charged carriers: $\mathrm{Sn}(\mathrm{IV}) \mathrm{TPPCl} \mathrm{P}_{2}$

In a recent paper, the interaction of Sn(IV) tetraphenylporphyrin with salicylate has been studied in detail. ${ }^{8}$ It was concluded that Sn(IV)TPP ${ }^{2+}$ indeed acts as an electrically charged carrier which selectively binds to two salicylate anions. Therefore, this ionophore should behave as illustrated schematically in Fig. 3F, and the incorporation of anionic additives could have a beneficial effect on the membrane's anion response. To investigate this effect, the potentiometric selectivity of different $o$-NPOE/PVC $(2: 1)$ membranes containing this ionophore and tridodecylmethylammonium chloride (TDMACl), potassium tetrakis $\{$ bis $(m$ trifluoromethyl)phenyl\}borate (KTFPB), or no additive at all was determined. As shown in Table 1, the selectivity of membranes containing Sn(IV)TPP2+ and TDMACl or TDMACl alone corresponds to the so-called Hofmeister series (see equation 5). The selectivity for membranes containing KTFPB as an additive clearly yield improved salicylate selectivity when compared to the one containing no anionic additives $(0 \%)$. These results clearly suggest that the incorporation of anionic additives does not lead to a deterioration of the membrane's potentiometric anion response as suggested earlier, ${ }^{6}$ and that Sn(IV)TPP ${ }^{2+}$ can be regarded as a charged carrier ionophore operating via the mechanism shown in Fig. 3F. It is important to note, however, that the membrane containing no additives already exhibits better selectivity toward salicylate than predicted by equation 4 . This implies that a substantial number of anionic sites are already present in the PVC membrane from endogenous impurities in the polymer. This would lead to an enhanced selectivity of the membrane compared to that expected based on the scenario shown in Fig. 3E. Indeed, the presence of anionic impurities in PVC has been confirmed previously, ${ }^{13,14}$ and has been linked to the very useful potentiometric response of neutral carrier-based cation-selective electrodes even when no exogenous anionic species are added to the membrane formulations (e.g. potassium sensors based on valinomycin, etc.).

It should be noted that the slopes of the $\mathrm{Sn}(\mathrm{TPP})^{2+}$-based electrodes toward salicylate were generally non-Nernstian $(-55 \mathrm{mV} / \mathrm{dec}$ for $0 \% \mathrm{R}^{-}, \quad-93 \mathrm{mV} / \mathrm{dec}$ for $10 \% \mathrm{R}^{-}, \quad-73$ $\mathrm{mV} / \mathrm{dec}$ for $20 \% \mathrm{R}^{-}$and $30 \% \mathrm{R}^{-}$) over the salicylate concentration range of $10^{-4}$ to $10^{-1} \mathrm{M}$. The origin of such super-Nernstian behavior is not yet known. Thus, the selectivity data reported in Table 1 have to be interpreted with some care since these values were determined using the theoretical slope of $-59.2 \mathrm{mV} / \mathrm{dec}$ via the separate solution method. ${ }^{21}$ Nonetheless, the differences in selectivity between adding lipophilic anionic $v s$. cationic sites appears to be real and significant, especially for lipophilic interfering anions (i.e. beyond that expected by taking into account the differences in slope).

Metal(III)porphyrins which may act as electrically charged or neutral ionophores: In(III)OEPCl and Co(III)TPPCl

In contrast to $\mathrm{Sn}(\mathrm{IV})[\mathrm{TPP}]^{2+}$, metal(III) porphyrins may act both as neutral or charged carriers, depending on the number of coordinating anionic ligands (see Fig. 1). If the fifth ligand is neutral (such as water), a charged carrier mechanism is expected. If both axial ligands are anionic, the ionophore may behave as neutral carrier. Moreover, compounds might exist which could act as both, depending on the nature of ionic additive and axial ligand present in the membrane. Therefore, the incorporation of anionic or cationic additives in membranes may elucidate such effects in more detail. As an 
Table 2. Selectivity coefficients of $o-N P O E / P V C ~(2: 1)$ membranes doped with $\ln (\mathrm{III})[\mathrm{OEP}] \mathrm{Cl}$ and different concentrations of ionic additives

\begin{tabular}{lccccccc}
\hline \multicolumn{7}{c}{$\log k$ Gi, } \\
$\mathbf{Y}^{-}$ & $30 \% \mathbf{R}^{-*}$ & $19 \% \mathbf{R}^{-*}$ & $9 \% \mathbf{R}^{-*}$ & $0 \% *$ & $11 \% \mathbf{R}^{+*}$ & $21 \% \mathbf{R}^{+*}$ & TDMACl ${ }^{*}$ \\
\cline { 2 - 7 } $\mathrm{NO}_{2}^{-}$ & 0.3 & 0.8 & 0.7 & 0.7 & 0.6 & 0.9 & 0.9 \\
$\mathrm{NO}_{3}^{-}$ & -3.8 & -4.1 & -3.8 & -3.2 & 1.0 & 1.4 & 1.7 \\
$\mathrm{Sal}^{-}$ & 2.2 & 2.7 & 2.7 & 1.9 & 2.5 & 2.7 & 3.3 \\
$\mathrm{SCN}^{-}$ & 1.4 & 1.3 & 1.2 & 1.5 & 3.6 & 3.9 & 3.3 \\
$\mathrm{I}^{-}$ & 0.9 & 0.7 & 0.7 & 1.2 & 3.6 & 3.3 & 3.3 \\
$\mathrm{ClO}_{4}^{-}$ & -2.6 & -2.5 & -1.9 & -0.7 & 4.5 & 4.8 & 4.7 \\
\hline
\end{tabular}

*Percentage given: molar amount of $\mathrm{KTFPB}\left(\mathrm{R}^{-}\right)$or $\mathrm{TDMACl}\left(\mathrm{R}^{+}\right)$relative to the total ionophore concentration in the membrane.

+Membrane with $1 \mathrm{wt} \%$ TDMACl (without ionophore).

example, the selectivity of In(III)[OEP ${ }^{+}$-based anion-selective electrodes in $o$-NPOE/PVC (2:1) incorporating different ionic additives is shown in Table 2. Based on the data presented, a charged carrier mechanism appears to exist, since the membrane with added TDMACl exhibits a Hofmeister type selectivity pattern. As expected, with TDMACl the electrode function showed no slope at all toward chloride, but exhibited anionic response for more lipophilic anions. Therefore, the coordination of two anionic ligands is obviously not favored by the In(III) metal center and, in this case, uncomplexed anions are extracted from the sample when the membranes contain added cationic sites (see Fig. 3F). On the other hand, the In(III)[OEP] ${ }^{+}$membranes containing KTFPB clearly show a significant change in selectivity compared with ones prepared without anionic additive. While the measured slopes toward chloride are again super-Nernstian $(-80$ $\mathrm{mV} / \mathrm{dec}$ for $0 \% \mathrm{R}^{-},-106.3 \mathrm{mV} / \mathrm{dec}$ for $9 \% \mathbf{R}^{-}$ and $19 \% \mathrm{R}^{-}$, and $-93.2 \mathrm{mV} / \mathrm{dec}$ for $30 \% \mathbf{R}^{-}$), the discrimination of lipophilic anions such as
$\mathrm{ClO}_{4}^{-}$and $\mathrm{I}^{-}$is clearly improved when the borate derivative is present in the membrane phase.

In the case of $\mathrm{Co}$ (III) porphyrins, the situation is quite different. The first highly anion-selective charged carrier reported in the literature was a lipophilic heptaester of Co(III) aquocyanocobyrinate, a derivative of vitamin $B_{12} .^{15,19}$ This ionophore was found to induce a high selectivity toward nitrite and thiocyanate. The chelating corrin ring is structurally related to the porphin ring, but the overall charge of the unprotonated/uncomplexed corrin ring is only -1 , as compared to -2 for the porphin. These structures are illustrated in Fig. 4 for the respective $\mathrm{Co}$ (III) complexes. This distinct difference is likely to induce a different mechanism for the potentiometric anion response of the $\mathrm{Co}$ (III) corrin vs. Co(III) porphyrin systems. Recently, $\mathrm{Co}$ (III) aquocyanocobyrinate has been clearly identified as a charged carrier, showing optimum selectivity with $10-60 \mathrm{~mol} \% \mathrm{KTFPB}$ as anionic additive, while the presence of $50 \mathrm{~mol} \%$ TDMACl induced Hofmeister selectivity. ${ }^{15}$
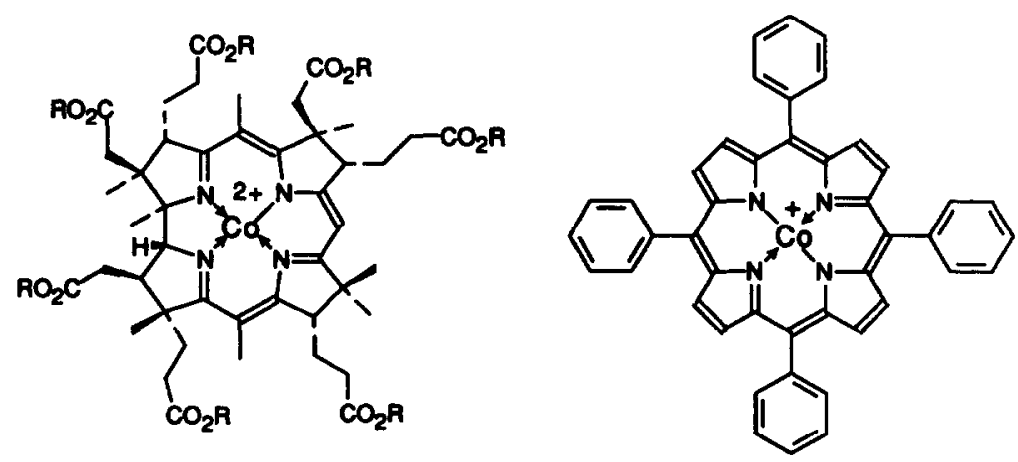

Fig. 4. Chemical structures of $\mathrm{Co}$ (III) cobyrinate and $\mathrm{Co}$ (III) tetraphenylporphyrin. Due to a difference in the net charge of the complexes, both compounds are expected to act differently in potentiometric membranes (see also Fig. 1). 


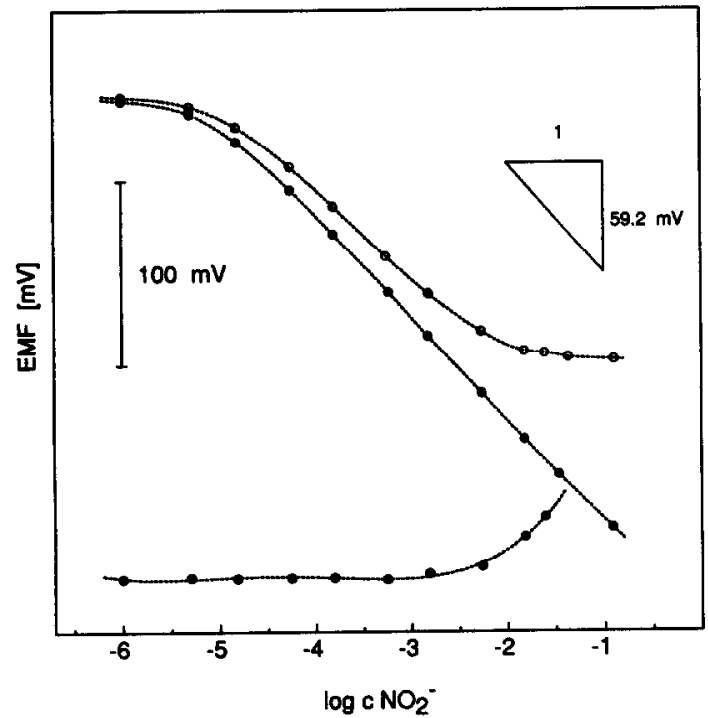

Fig. 5. Typical potentiometric responses toward nitrite (in MES buffer, pH 5.5) of membranes containing $\mathrm{Co}(\mathrm{III})[\mathrm{TPP}] \mathrm{Cl}$ and $(\oplus) 10 \mathrm{~mol} \% \mathrm{KTFPB},(O)$ no ionic additives, and (O) $10 \mathrm{~mol} \% \mathrm{TDMACl}$ in $o$-NPOE/PVC (2:1).

Considering the difference in the charge of the porphyrin ring, it may be expected that $\mathrm{Co}$ (III) tetraphenylporphyrin can act as an electrically neutral carrier, since the overall charge of the complex coordinated to two anionic axial ligands would be -1 instead of zero for the corrin system.

To gain more insight into the mechanism of $\mathrm{Co}(\mathrm{III})[\mathrm{TPP}]^{+}$derivatives, we prepared $o$ NPOE/PVC (2:1) membranes containing $\mathrm{Co}(\mathrm{III})[\mathrm{TPP}] \mathrm{Cl}$ in the presence of various ionic additives. Neutral axial ligands (e.g. pyridine $e^{4,12}$ ) were omitted in this stage for simplicity. The membranes were conditioned in $0.01 M \mathrm{NaNO}_{2}$ prior to measurement, and the ligand exchange at the metal center from $\mathrm{Cl}^{-}$to $\mathrm{NO}_{2}^{-}$was confirmed spectrophotometrically according to a method described elsewhere ${ }^{12}$ (data not shown). As shown in Fig. 5, only the slopes for the membranes containing cationic or no additives were found to be anionic. The slope for the membrane containing KTFPB was clearly cationic, indicating that a neutral carrier mechanism is taking place (see Fig. 1B). For freshly prepared membranes without additives, the observed anionic slope of $-47 \mathrm{mV} / \mathrm{dec}$ is somewhat surprising (see also Ref. 3), but after only 4 days, the response towards nitrite was found to dramatically decrease $(-23 \mathrm{mV} / \mathrm{dec})$, whereas the slopes of the membranes containing TDMACl were, for the most part, nearly theoretical ( $-57 \mathrm{mV} / \mathrm{dec}$ for $10 \%$ and $26 \% \mathrm{R}^{+},-60$ $\mathrm{mV} / \mathrm{dec}$ for $39 \%$ and $52 \% \mathrm{R}^{+}$, and -26 $\mathrm{mV} / \mathrm{dec}$ for $80 \% \mathrm{R}^{+}$) and remained constant over a period of at least 2 weeks (data not shown). Hence, analytically useful electrode membranes with $\mathrm{Co}$ (III) tetraphenylporphyrin ionophore can only be achieved with the simultaneous addition of lipophilic cationic sites such as TDMACl. As expected based on equation 2 and Fig. 2, optimal selectivity for nitrite over bulky interfering ions such as perchlorate was achieved with lower concentrations of TDMACl (see Table 3). In general, however, membranes with up to $50 \mathrm{~mol} \%$ cationic additives showed nearly equal potentiometric selectivity behavior. Only the membrane containing a high level ( $80 \mathrm{~mol} \%$ ) of TDMACl begins to approach the Hofmeister sequence. As expected, membranes containing the $\mathrm{Co}$ (III) porphyrin ionophore in their optimized formulation exhibit the same general preference toward nitrite (and thiocyanate) as the previously reported $\mathrm{Co}$ (III) aquocyanocobyrinate derivatives. ${ }^{15}$ At this stage, however, the more lipophilic anions (e.g. perchlorate, salicylate, etc.) seem to be more discriminated against with the cobyrinate compound than with the tetra-

Table 3. Selectivity coefficients of $o$-NPOE/PVC $(2: 1)$ membranes doped with $\mathrm{Co}$ (III)[TPP]Cl and different concentrations of TDMACl as cationic additive

\begin{tabular}{lrrrrrrr}
\hline & \multicolumn{7}{c}{$\log k \mathrm{NO}_{2, Y}^{\text {pot }}$} \\
\cline { 2 - 8 } $\mathrm{Y}^{-}$ & $0^{*} \%^{*}$ & $10 \% *$ & $26 \% *$ & $39 \% *$ & $52 \% *$ & $80 \% *$ & TDMACl $\%^{*}$ \\
\hline $\mathrm{Cl}^{-}$ & -1.9 & -3.2 & -3.3 & -3.6 & -3.6 & -1.7 & -0.9 \\
$\mathrm{NO}_{3}^{-}$ & -1.8 & -2.2 & -2.3 & -2.1 & -2.1 & 0.0 & 0.8 \\
$\mathrm{Sal}^{-}$ & -0.5 & -0.9 & -0.9 & -0.7 & -0.7 & 1.2 & 2.4 \\
$\mathrm{SCN}^{-}$ & 0.8 & 1.0 & 1.0 & 1.1 & 1.1 & 1.7 & 2.4 \\
$\mathrm{ClO}_{4}^{-}$ & -0.4 & 0.1 & 0.3 & 0.6 & 0.6 & 2.7 & 3.8 \\
\hline
\end{tabular}

*Percentage given: molar amount of TDMACl relative to the total ionophore concentration in the membrane.

†Membrane with $1 \mathrm{wt} \%$ TDMACl (without ionophore). 
phenylporphyrin derivative used here. However, the nature of the fifth axial ligand may be important for the binding strength of the metal center towards the analyte anion as the sixth ligation site. In this respect, the fifth ligand $\mathrm{CN}^{-}$ for the cobyrinate compound is likely to induce a different selectivity than the $\mathrm{NO}_{2}^{-}$in the system examined here. Moreover, $\mathrm{Co}$ (III) porphyrins reported in the literature for use in anion-selective electrodes were usually coordinated to one neutral axial ligand such as pyridine ${ }^{4,12}$ and, therefore, even a charged carrier mechanism may be proposed in these cases, if the neutral ligand is not displaced by the analyte anion when conditioning the electrode in nitrite solutions prior to use. The evaluation of such differences is currently in progress.

\section{CONCLUSIONS AND FUTURE DIRECTIONS}

It has been shown that the incorporation of lipophilic anionic or cationic additives can significantly change the selectivity of anion-selective electrodes based on metalloporphyrins. While $\mathrm{Sn}(\mathrm{IV})[\mathrm{TPP}]^{2+}$ has been identified as charged carrier, the metal(III) porphyrins can either function via a neutral or charged carrier mechanism, depending on the number of anionic ligands coordinated to the metal center. While In(III)[OEP]Cl exhibits distinct selectivity toward chloride in membranes containing $\mathrm{KTFPB}, \mathrm{Co}$ (III)[TPP]Cl induces a cationic electrode function with KTFPB, and only shows good selectivity towards nitrite and thiocyanate in the presence of the cationic additive TDMACl. Hence, a neutral carrier mechanism for $\mathrm{Co}(\mathrm{III})[\mathrm{TPP}]^{+}$can be assumed in this case. The theoretical and experimental findings reported above suggest a number of new research directions for the future. These include: (a) re-examining metalloporphyrins previously screened as possible ionophores in anion-selective electrodes in membranes now containing lipophilic ionic sites [especially metal(II) and metal(III) porphyrins which might actually function via a neutral carrier mechanism]; (b) examining other membrane materials such as silicone rubber or polyurethane to determine how endogenous anion impurities of given polymers can influence the initial selectivities observed without the incorporation of exogenous additives and (c) the evaluation of potentiometric selectivities of metalloporphyrins incorporated into pure liquid solvents (i.e. U-Tube experiments) to ascertain the true response function of the metallopor- phyrins in the absence and presence of known amounts of charged additives without concomitant effects from unknown ionic impurities already present in most polymer matrices. In addition, it will be necessary to further understand the chemistry that leads to the superNernstian behavior of membranes doped with Sn(IV) and In(III) porphyrins, especially the role of plasticizer dielectric constant on such non-theoretical responses. Clearly, the proposed work in pure solvents without endogenous contaminants will help in unravelling this mystery. Such studies are currently in progress in this laboratory.

Acknowledgements-We gratefully acknowledge the National Institutes of Health (GM-28882), the Swiss National Science Foundation, and the State Committee for Scientific Research (Poland) for supporting this work.

\section{REFERENCES}

1. Q. Chang, S. B. Park, D. Kliza, G. S. Cha, H. Yim and M. E. Meyerhoff, Am. Biotech. Lab., 1990, 8, 10.

2. V. J. Wotring, D. M. Johnson, S. Daunert and L. G. Bachas, in Immunochemical Assays and Biosensor Technology for the 1990's, R. M. Nakamura, Y. Kasahara and G. A. Rechnitz (eds), pp. 355-376. American Society of Microbiology, 1992.

3. M. Rothmaier and W. Simon, Anal. Chim. Acta, 1993, 271, 135.

4. D. Ammann, M. Huser, B. Kräutler, B. Rusterholz, P. Schulthess, B. Lindemann, E. Halder and W. Simon, Helv. Chim. Acta, 1986, 69, 849.

5. N. A. Chaniotakis, A. M. Chasser, M. E. Meyerhoff and J. T. Groves, Anal. Chem., 1988, 60, 188.

6. A. Jyo, and H. Egawa, Anal. Sci., 1992, 8, 823.

7. N. A. Chaniotakis, S. B. Park and M. E. Meyerhoff, Anal. Chem., 1989, 61, 566.

8. C. E. Kibbey, S. B. Park, G. Deadwyler and M. E. Meyerhoff, J. Electroanal. Chem., 1992, 335, 135.

9. S. B. Park, W. Matuszewski, M. E. Meyerhoff, Y. H. Liu and K. M. Kadish, Electroanalysis, 1991, 3, 909.

10. M. Huser, W. E. Morf, K. Fluri, K. Seiler, P. Schulthess and W. Simon, Helv. Chim. Acta, 1990, 73, 1481.

11. A. Hodinár and A. Jyo, Chem. Lett., 1988, 993.

12. X. Li and D. J. Harrison, Anal. Chem., 1991, 63, 2168.

13. E. Lindner, E. Graf, Z. Niegreisz, K. Tóth, E. Pungor and R. P. Buck, Anal. Chem., 1988, 60, 295.

14. A. van den Berg, P. D. van der Wal, M. SkowronskaPtasinska, E. J. R. Sudhölter, D. N. Reinhoudt and P. Bergveld, Anal. Chem., 1987, 59, 2827.

15. U. Schaller, E. Bakker, U. E. Spichiger and E. Pretsch, Anal. Chem., 1994 66, 391.

16. J. W. S. Buchler, The Porphyrins, D. Dolphin (ed.), Academic Press, New York, 1978.

17. H. Sugimoto, N. Ueda and M. Mori, Bull. Chem. Soc. Jpn, 1981, 54, 3425.

18. T. Sakurai, K. Yamamoto, H. Naito and N. Nakamoto, Bull. Chem. Soc. Jpn. 1976, 49, 3042.

19. P. Schulthess, D. Ammann, W. Simon, C. Caderas, R. 
Stepánek and B. Kräutler, Helv. Chim. Acta, 1984, 67, 22. T. Rosatzin, E. Bakker, K. Suzuki and W. Simon, Anal. 1026. Chim. Acta, 1993, $280,197$.

20. M. Telting-Diaz, M. E. Collison and M. E. Meyerhoff, Anal. Chem., 1994 66, 576.

21. G. G. Guilbault, R. A. Durst, M. S. Frant, H. Freiser, E. H. Hansen, T. S. Light, E. Pungor, G. Rechnitz, N. M. Rice, T. J. Rohm, W. Simon and J. D. R. Thomas, Pure Appl. Chem., 1976, 48, 127.

23. W. E. Morf, The Principles of Ion-Selective Electrodes and of Membrane Transport, in E. Pungor, W. Simon, J. Inczédy (eds), Studies in Analytical Chemistry, Vol. 2. Akadémiai Kiadó, Budapest, 1981.

24. R. P. Buck, V. V. Cosofret and E. Lindner, Anal. Chim. Acta, 1993, 282, 273. 